\title{
Revision total knee arthroplasty: hybrid vs standard cemented fixation
}

\author{
Jesús Gómez-Vallejo*, Jorge Albareda-Albareda, Belén Seral-García, Nieves Blanco-Rubio \\ and Laura Ezquerra-Herrando
}

\begin{abstract}
Introduction: Modular systems with stems are necessary for the stability of revision total knee arthroplasty (rTKA), but controversy remains as to the best fixation method: cemented or hybrid (noncemented stem). The aim of this study was to assess the clinical, X-ray, life-quality and survival results obtained with each fixation method.

Materials and methods: During the period 2000-2013, rTKA was performed on 67 patients (29 cemented arthroplasty and 38 hybrid fixation). The average follow-up was 7 years (range 2-15). All patients were evaluated clinically and radiographically using the American Knee Society Score (AKSS), the Western Ontario and McMaster Universities Arthritis Index (WOMAC), and the Short Form Health Survey (SF-36). A survival study was performed via Kaplan-Meier analysis.

Results: There were no differences between the cemented and hybrid fixation groups in the preoperative and postoperative AKSS clinical evaluation indices and the SF-36 health index. However, the WOMAC assessment scale did reveal statistically significant differences between the groups, with a global classification of 64.9 points weighted at 100 (SD 16.8) for cemented fixation versus 78.9 (SD 9.0) for hybrid fixation $(p=0.001)$. The corresponding values for stiffness were 61.6 (SD 12.9) and 80.5 (SD 14.7) ( $p=0.001$ ), and those for function were 61.3 (SD 19.4) and 78.1 (SD 10.5) $(p=0.001)$. No significant differences between the groups were recorded with respect to the pain score $(p=0.4)$ or the results of the Kaplan-Meier survival analysis.
\end{abstract}

Conclusion: Although the results were similar for the two groups, hybrid fixation tended to produce better results than cemented fixation. In view of the risk of further loosening, we prefer the more conservative approach, i.e. hybrid fixation.

Level evidence: Level III.

Keywords: Revision knee arthroplasty, Stem, Cemented fixation, Hybrid fixation

\section{Introduction}

Revision arthroplasty of the knee is one of the great challenges in orthopaedic surgery. The number of prosthesis replacements performed is rising, but the surgical procedure is very demanding: there is very little bone substrate and its architectural quality is poor [1]. In response, modular revision prostheses are viewed as an excellent treatment option [2]. The surgical material used in this type of surgery must be very versatile, as total knee revision

*Correspondence: jgomezvallejo@yahoo.es

Department of Orthopedic Surgery, "Lozano Blesa" University Hospital, Joaquina Zamora 4, 4 B, 50018 Saragossa, Spain arthroplasty poses several very complex problems, especially bone loss and insufficient soft tissue. The use of metal wedges and augmentations to compensate for bone loss has become generally accepted, and morselised or structural bone grafts are also commonly employed to treat bone defects [3].

Constrained prostheses are widely used to overcome the lack of ligaments. However, the constriction system requires the contact surface between bone and prosthesis to withstand a large number of force vectors, and therefore the replacement surface must be cemented [1]. Nevertheless, modular systems with stems are now widely used for strengthening prosthesis stability. 
The question then arises: is it actually necessary to cement these central-medullary devices? Cemented stems have been shown to perform well and to be long lasting. However, there are drawbacks, especially the stress shielding that may occur and the difficulty involved in removing the cement if further revision is necessary [4]. Several papers have addressed this question, but few have compared the two systems, and fewer still have employed a life-quality index.

The primary aim of the study reported in the present paper was to assess how the clinical and life-quality outcomes achieved depend on the fixation method employed (hybrid or cemented) in total knee arthroplasty replacement. Secondary outcome measures were survival and $\mathrm{X}$-ray results. The hypothesis of this study was that the hybrid fixation method achieves better clinical and X-ray results than the cemented stem method.

\section{Materials and methods}

The study performed was analytical and retrospective. The study population was obtained by reference to the database maintained at the authors' institution. The patients selected for study were consecutively operated on from January 2000 to December 2013 for revision total knee arthroplasty (rTKA), including either cemented stem (Natural Knee II, Centerpulse ${ }^{\circledR}$ Warsaw, IN, USA) or hybrid fixation (P.F.C. TC-3 Sigma, DePuy ${ }^{\circledR}$ Raynham, MA, USA). The implant decision was taken by a single surgeon. 97 cases were considered for analysis, imposing the following inclusion criteria: surgery performed at the abovementioned institution in the period January 2000 to December 2013, due to failure of the primary prosthesis; revision surgery performed on only one knee; replacement recommended due to aseptic failure; at least the tibial and femoral components were replaced in this surgery. The exclusion criteria were: septic loosening of the previous implant; prior replacement surgery on the same knee, in any form; failure to provide informed consent to participate in the study. Of the original population, 21 were lost to follow-up, and nine were excluded due to septic loosening of the implant.

Finally, therefore, a sample group of 67 patients was formed, of whom 29 received a cemented arthroplasty and 38 a hybrid fixation arthroplasty. The choice of implant was decided at the surgeon's discretion, without randomisation.

All these revision surgeries were performed by the same physician (FS). In every case, the bone cement was used without antibiotic and systematic cephazolin was provided.

Low-molecular-weight heparin was given the evening before surgery and then once daily for 1 month. Ambulation began on the second postoperative day with weight bearing as tolerated with canes. Physical therapy included daily range-of-motion exercises, assisted by continuous passive-motion machines. Patients continued physical therapy as outpatients after hospital discharge.

The average follow-up of the revision arthroplasties was 7 years (range 2-15; SD: 3 years). All patients were evaluated prior to surgery.

The epidemiological data collected included age, gender, body mass index and years elapsed since the primary arthroplasty. In addition, the ASA Physical Classification System was applied [5] and bone status was determined according to the Anderson Orthopaedic Research Institute Classification [6].

The following surgery data were recorded: type of procedure, haemorrhage during procedure, duration of surgery and size of insert.

The American Knee Society Score (AKSS) [7] was used to assess the joint, both prior to surgery and at the final postoperative follow up. In the latter examination, the Western Ontario and McMaster Universities Arthritis Index (WOMAC) [8] and the Short Form Health Survey (SF-36) [9] index were also determined. The scores for these two indices were weighted at 100 , where 0 is the worst possible result and 100 the best. The AKSS, WOMAC and SF-36 indices were taken as the primary outcome measures.

The modified AKSS proposed by Fehring [10] was used to perform the evaluation of the final X-ray image obtained. To facilitate analysis of the stem, the implant was divided for study into specific areas: 14 for the femur and 16 for the tibia (see Fig. 1). The radiolucency lines were measured in millimetres in each area, and are shown according to the implant performed. For the femur, an implant was considered stable if the total thickness of these radiolucency lines was no more than $8 \mathrm{~mm}$; from 9 to $19 \mathrm{~mm}$, monitoring was required, and $20 \mathrm{~mm}$ or more reflected the existence of a loose implant. On the tibia, the corresponding values were $\leq 9,10-22$ and $\geq 23 \mathrm{~mm}$.

An implant was considered to have failed when the patient subsequently required a revision of any of the prosthetic components or was on the surgical waiting list for the same reason. In our study, six of the implants were classed as loose: four were cemented and two were hybrid fixations.

With respect to the primary outcome measures, the Student $t$ test was used to compare the quantitative variables that followed a normal distribution with the dichotomous variables (hybrid vs. cemented). The Kolmogorov-Smirnov test was applied to determine whether quantitative variables were normally distributed. The Pearson correlation test was used to verify the relationship between two quantitative variables. In all cases, 

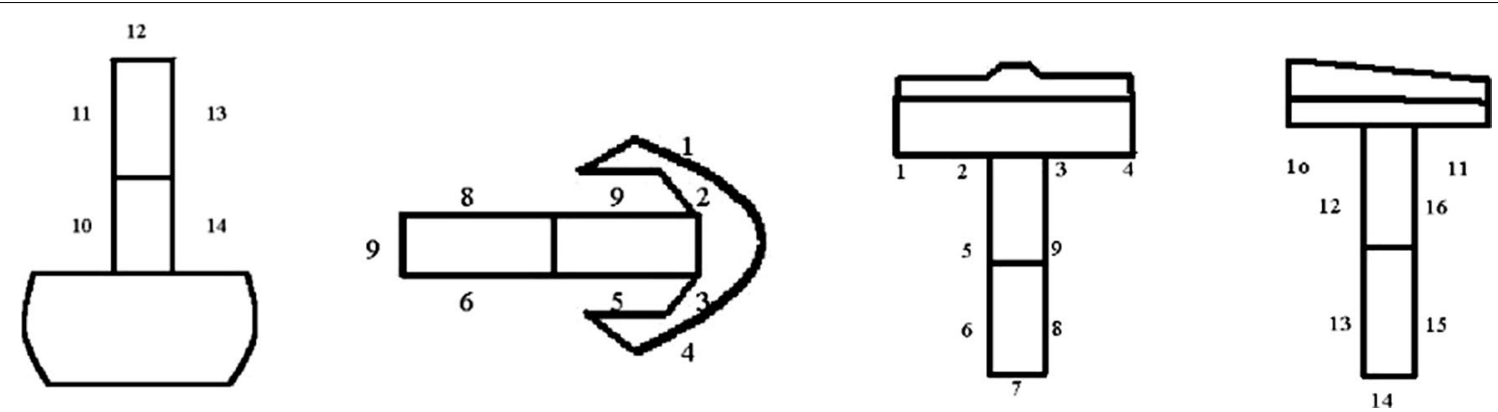

Fig. 1 Areas in the Fehring modified AKSS X-ray evaluation

Table 1 AKSS and SF-36 results for the cemented (c) and hybrid (h) arthroplasties

\begin{tabular}{lllll}
\hline & Type & $\boldsymbol{N}$ & Mean & $\begin{array}{l}\text { Standard } \\
\text { deviation }\end{array}$ \\
\hline AKSS function $(p=0.12)$ & c & 29 & 77.80 & 11.87 \\
AKSS $(p=0.82)$ & h & 38 & 72.26 & 16.17 \\
SF physical $(p=0.32)$ & c & 29 & 70.65 & 18.04 \\
SF mental $(p=0.14)$ & h & 38 & 71.65 & 19.08 \\
& C & 29 & 60.99 & 13.42 \\
SF-36 $(p=0.11)$ & h & 38 & 64.70 & 16.13 \\
& h & 29 & 66.19 & 11.51 \\
& c & 29 & 70.83 & 13.50 \\
& h & 38 & 69.32 & 13.28 \\
\hline
\end{tabular}

95\% confidence intervals were calculated. For the secondary outcome measures, the chi-square test was used to compare the qualitative variables. The survival study was performed by Kaplan-Meier analysis, with statistical significance assumed at $p<0.05$.

\section{Results}

The preoperative and postoperative AKSS clinical evaluation indices and the SF-36 health index revealed no differences by cementation type (Table 1 ).

The presence of pain, according to the AKSS scale, also did not differ statistically significantly between the cemented and hybrid fixation groups. In the hybrid-fixation group, there were only three cases $(8 \%)$ of pain at the tip of the shaft in the tibia, and the number of such cases in the cemented group was not statistically significant either.

The average postoperative flexion was $89^{\circ}$ (SD 17) among the cemented arthroplasties and $90^{\circ}$ (SD 20) among the cases of hybrid fixation. For extension, a statistically significant difference was found $\left(3^{\circ}, \mathrm{SD} 6\right.$ vs $1^{\circ}$, SD 3) favouring cemented fixation $(p=0.04)$. Joint balance was not statistically significant between the groups $\left(88.2^{\circ}\right.$, SD 17.3 in the cemented-fixation group and $86.8^{\circ}$, SD 23.4, in the hybrid-arthroplasty group; $p=0.74$ ).

The WOMAC assessment scale did present statistically significant differences in global classification (64.9 points weighted at 100 for the cemented arthroplasties, SD 16.8; and 78.9, SD 9.0, for the hybrid ones; $p=0.001$ : 95\% CI 7.62-20.38), stiffness (61.6, SD 12.9; and 80.5, SD 14.7; $p=0.001: 95 \%$ CI 12.03-25.77) and function (61.3, SD 19.4, and 78.1, SD 10.5 ( $p=0.001$ : 95\% CI 9.42-24.18). However, no such differences between the groups were observed for the pain score $(p=0.4)$.

No differences between the groups were found in the pre- to postoperative variations of the AKSS index ( $p=0.63$ joint scoring and $p=0.09$ functional scoring) and joint balance $(p=0.79)$. We conclude, therefore, that the improvement achieved was the same for both types of prosthesis fixation (Fig. 2).

No statistically significant differences between the treatment groups were found for age, gender, ASA or body mass index (Table 2).

Similarly, there were no significant differences between the groups in the operation variables. The surgery time was practically the same (143 $\mathrm{min}$ for both cemented and hybrid fixations, SD 23.5 and 23.7, respectively). The amount of bleeding was slightly higher in the hybrid fixation arthroplasties, where the tourniquet was removed before closing the joint (308 cc, SD 137 vs 282 cc, SD 211 in the cemented fixations). There were no differences between the groups regarding the use of bone grafts or augmentations, or in the Anderson classification (mode $2 \mathrm{~A}$ for the tibia and $2 \mathrm{~B}$ for the femur). For the cemented arthroplasties, the mode value for the length of the stem in the femur was $125 \mathrm{~mm}(80 \%)$, and for the diameter it was $10.5 \mathrm{~mm}$. The most commonly used types of stem in the tibia measured $60 \mathrm{~mm}$ and were modular and conical. In the hybrid fixation group, the mode value for the length of the femur component was $125 \mathrm{~mm}$ and for the diameter it was $14 \mathrm{~mm}$, while the respective values for the tibia were 115 and $12 \mathrm{~mm}$. 


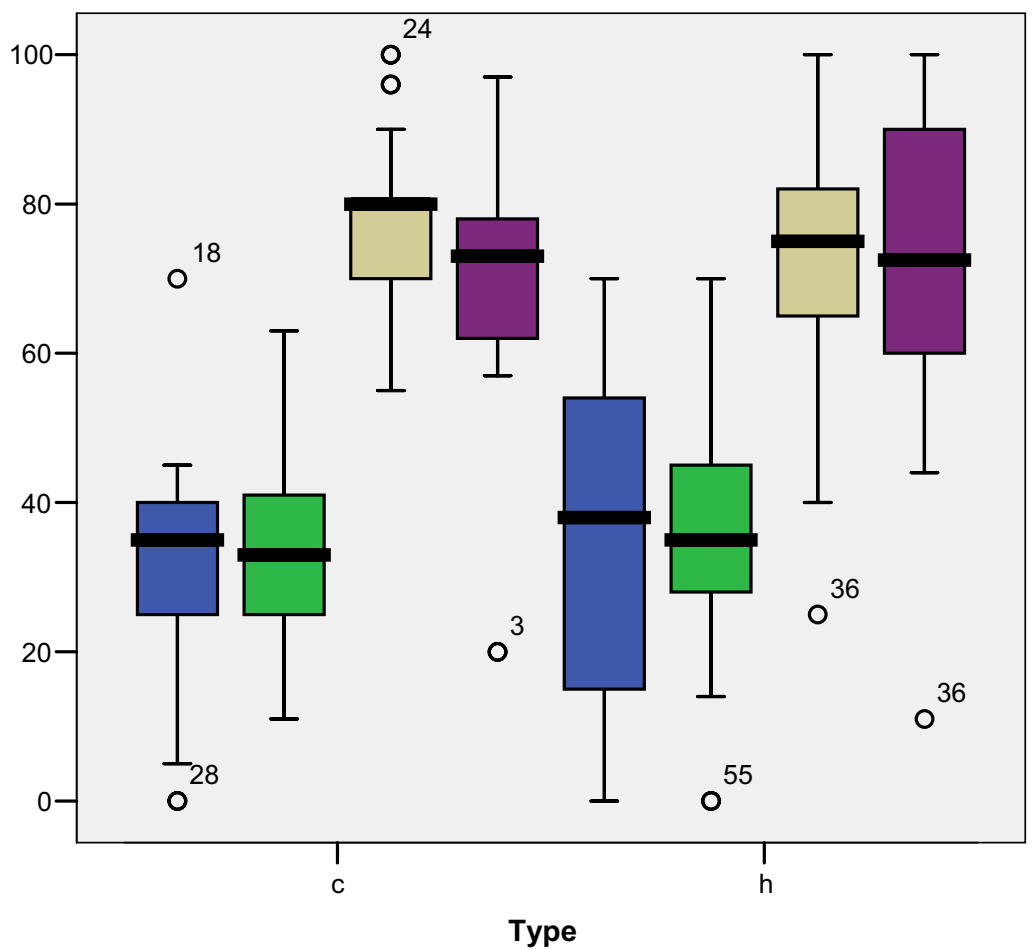

$\square$ KSS functional PRE

KSS joint PRE

KSS functional POST

KSS joint POST

Type

Fig. 2 Group comparison [cemented (c) and hybrid ( $h$ )] of the pre- to postoperative variation in the AKSS index

Table 2 BMI and age results for cemented (c) and hybrid (h) arthroplasties

\begin{tabular}{lllll}
\hline & Type & $\boldsymbol{N}$ & Mean & Standard deviation \\
\hline BMl & c & 29 & 31.1138 & 3.69040 \\
& h & 38 & 31.2158 & 4.43651 \\
Age & c & 29 & 79.7241 & 4.37441 \\
& h & 38 & 78.3684 & 4.43220
\end{tabular}

The patients were followed up at 4 weeks after the operation and again after 2, 4, 6 and 12 months. Subsequent follow-ups were yearly, and anteroposterior and lateral X-rays were taken on each occasion.

Assessment of the radiological stability of the implants did not reveal any significant variations, with a mean value of 2 (range $0-19$ ) for the cemented femurs and 3 (range $0-12$ ) for the hybrid fixations $(p=0.22)$. According to the Fehring classification, the mean value was $4(0-12)$ for the cemented tibias and $3(0-10)$ for the hybrid fixations $(p=0.15)$. Among the cemented fixations, four implants had possibly loosened in the tibia and two in the femur. No case was defined as migrated. Among the hybrid fixations, there was one possibly loose implant in the tibia and four in the femur. Here, too, there were no migrations.
Six of the arthroplasties were classed as failed-three due to late-onset infection, one because of femoral stem breakage caused by fatigue after 6 years of evolution in a cemented prosthesis, and two due to instability.

Regarding the survival of the implants, using any rerevision as the endpoint, no statistically significant difference between the groups was found. Over time, both curves were similar: at 2 years $(100 \%$ in the cemented-fixation group and 97\% (CI 93-101) in the hybrid-implant group), at 5 years (93\%, CI 83-103 and 94\%, CI 85-101) and at 10 years (84\%, CI 70-98 and 94\%, CI 86-102). In the cemented cases, the corresponding values were notably lower, but the difference did not reach statistical significance $(p=0.61)$ (Fig. 3$)$.

\section{Discussion}

In studies of revision arthroplasty, the type of fixation that should be employed is a question that has attracted much attention. However, very few papers have compared the two main types of fixation: cemented and hybrid. The main conclusion reached in the present study is that the two systems achieve similar clinical results and survival times.

The biomechanics of these implants have been studied in numerous experimental models. Thus, Completo 


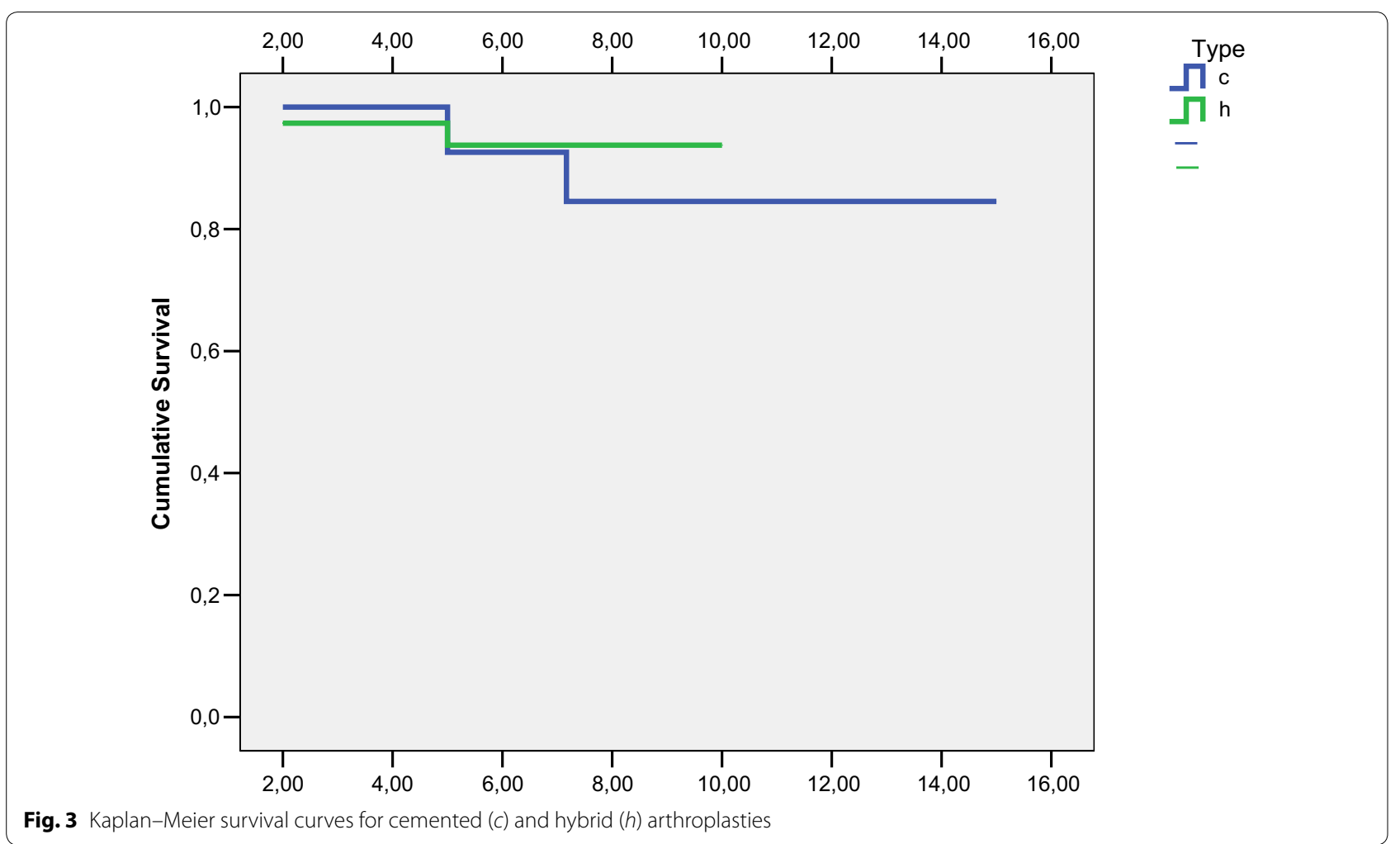

et al. $[11,12]$ studied the fixation of implants in vitro in revision implant surgery, analysing the behaviour of the proximal tibia using a synthetic model. Those authors reported that load distribution in the tibia was better with a long, noncemented stem. On the other hand, another study conducted in the same year to examine the behaviour of implants in tibia bone defects reached the conclusion that both types of fixation performed similarly in cavitary defects, although the cemented implant seemed to be the better option for large defects treated with structural allografts. However, in a clinical study of large-scale osteolysis, the authors recommended hybrid fixation, although this decision should be taken according to the bone quality observed (evidence level III) [13].

Jazrawi et al. [14] conducted a study, based on an examination of 12 cadavers, of the pressure vectors presented by cemented and hybrid stems, and concluded that with cemented stems there is less micromovement of the implant but increased stress shielding on the proximal tibia. Therefore, for an impacted stem to achieve a fixation as stable as that provided by a cemented implant, it must be larger (specifically, $75-150 \mathrm{~mm}$ ).

Skawara et al. [15] studied cadavers subjected to cyclical loads. Radiostereometric analysis showed that the load distribution was more regular in hybrid fixations and failed less often than in cemented implants. Accordingly, those authors recommended the former approach, although it should be noted that the fixations they studied were primary implants. In summary, the findings of those studies are inconclusive, and both methods seem to obtain similar results.

Few studies have reported results obtained from implants with stems for knee arthroplasty revision, and most of those studies are retrospective [16].

Some papers have discussed cemented and hybrid revision arthroplasties, but without performing a direct comparison of the results obtained $[17,18]$. Thus, Kim et al. analysed surgical interventions-cemented or hybrid fixations-performed on 114 knees and calculated the WOMAC score before and after surgery (15 and 65.5, respectively, vs the global postoperative score of 72.3 obtained in our analysis), but without differentiating between types of fixation.

In our own study, there were no statistically significant differences regarding the AKSS and the SF-36 indices, although the results were better with hybrid fixation. There were differences between the groups in the WOMAC scores for function and stiffness, but not for pain. There were no differences regarding the improvement gained according to the AKSS and the measurements of joint flexion, extension and balance (before and after surgery). These findings suggest that both systems are appropriate and equally effective. This point highlights one of the weak areas of our study: we did not 
have access to preoperative WOMAC and SF-36 scores because this procedure was not initiated in our department until after the study began.

Cemented fixation has probably been less extensively studied than the hybrid approach, but more research in this area is now being undertaken $[19,20]$. In this respect, the poorest results are those reported by Whaley et al. [21], with an AKSS function score of 56.9 (from a basal value of 16) and a knee score of 51 (from a baseline of 48), and the best ones are those noted by Mabry et al. [22], with an AKSS function score of 85 and a knee score of 91 .

By contrast, many papers have praised the virtues of hybrid fixation [1, 23, 24], reporting good-to-excellent results and recommending this surgical method. The results obtained for functional AKSS range from 55 in the worst series to 82 in the best, and from 79 to 85 for joints [2, 25-29].

Following Vince and Longe [25], many advocates of cemented fixation have criticised the use of constrained implants with hybrid fixation for revision knee arthroplasty. However, the failures observed by these authors occurred in septic revisions, where there was more severe structural damage and outcomes were inevitably worse. Other, well-designed studies [17, 30-32] recommend the use of constrained prostheses such as TC-III, reporting very good results from this approach, and they reserve the cemented-stem method for hinged prostheses presenting considerable loss of bone structure.

Another factor that should be taken into account is that the range of variables considered in reports of hybrid revisions is much narrower than in those focusing on cemented fixations, which would make the former results appear more predictable.

According to our literature review, few studies have been undertaken to compare X-ray images by type of prosthesis fixation (cemented or hybrid). Among this limited body of research, Peters et al. [18] observed no differences according to the type of fixation, although there appeared to be more lucency around the metaphyseal area in the hybrid-fixation implants (performed using a different technique to that examined here), which was plausibly explained by the nonimpaction of cement in that area. Fehring et al. [10] examined a series of hybrid-fixation arthroplasties and reported that $71 \%$ were stable, $19 \%$ were possibly loose and $10 \%$ had migrated-values that were significantly different from those obtained for a corresponding cemented series, with $93 \%$ stable and $7 \%$ possibly loosened. However, as mentioned above, that study concerned the comparison of metaphyseal stem X-rays and did not address the question of survival, nor mention any clinical scale. Reported radiolucency rates are similar for both types of fixation, suggesting that the type of fixation employed does not affect the X-ray results obtained [33-36].

Our study revealed no significant differences in survival between the two types of fixation. Although there was a difference of $10 \%$ between the respective survival rates at 10 years, this difference was only $1 \%$ at 5 years.

In our literature review, we also considered the longevity of the implants, taking into account only those results obtained by Kaplan-Meier estimation or similar, without regard for the percentage of failures. In this respect, cemented arthroplasties were again the leastcommonly studied; only two papers addressed this question, reporting survival rates of $97 \%$ at 5 years and $89-94 \%$ at 10 years (in contrast to the $84 \%$ found in our series) $[18,19]$. For hybrid-fixation arthroplasties, the results were similar. The series were somewhat longer in time than those of the cemented fixations, and the values obtained ranged from 92 to $95 \%$ at 5 years ( $93.7 \%$ in our series of hybrid fixations) to $83-93.5 \%$ at 10 years (94\% in our series) [2, 18, 28, 29, 37, 38]. Sheng et al. [31] recorded greater longevity for cemented than for completely noncemented fixations. A final consideration is that the research in this field was basically carried out between 2000 and 2012, and so the outcome over the last decade remains to be established.

This study has certain limitations. The number of subjects required was not determined a priori. Moreover, the study sample is relatively small (although this is also the case in most of the studies reviewed). In addition, the study groups were not randomised, and this reduces the statistical power obtained.

Taking into consideration the data previously reported, together with the findings presented here, we conclude that there are no great differences, in practice, between the two types of fixation. Cemented and hybrid fixations both constitute reproducible, safe procedures. Nevertheless, in view of the possible need for further revisions, and taking into account the complexity of the removal of cement, our choice is to use hybrid stems.

\section{Authors' contributions}

$J G, J A, B S$ and NB initiated and planned the study, prepared the database, performed the statistical calculations and wrote the manuscript. LE helped prepare the manuscript. All authors read and approved the final manuscript.

\section{Competing interests}

The authors declare that they have no competing interests.

\section{Ethics approval and consent to participate}

The study conforms to the 1964 Helsinki Declaration and its later amendments, and was approved by the Ethics Committee of the Hospital Center. All patients gave informed prior consent for inclusion in the study.

Funding

The authors did not receive specific funding for this work. 


\section{Publisher's note}

Springer Nature remains neutral with regard to jurisdictional claims in published maps and institutional affiliations.

Received: 2 February 2017 Accepted: 25 January 2018

Published online: 17 August 2018

\section{References}

1. Bertin KC, Freeman MAR, Samuelson KM et al (1985) Stemmed revision arthroplasty for aseptic loosening of total knee arthroplasty. J Bone Jt Surg (Br) 67:242-248

2. Peters LC, Erickson J, Kloepper RG et al (2005) Revision total knee arthroplasty with modular components inserted with metaphyseal cement and stems without cement. J Arthroplasty 20:302-308

3. Engh GA, Herzwurm PJ, Parks NL (1997) Treatment of major defects of bone with bulk allografts and stemmed components during total knee arthroplasty. J Bone Jt Surg (Am) 79(7):1030-1039

4. Patel AR, Barlow B, Ranawat AS (2015) Stem length in revision total knee arthroplasty. Curr Rev Musculoskelet Med 8(4):407-412

5. Keats AS (1978) The ASA classification of physical status - a recapitulation. Anesthesiology 49(4):233-236

6. Engh GA, Ammeen DJ (1999) Bone loss with revision total knee arthroplasty: defect classification and alternatives for reconstruction. Instr Course Lect 48:167-175

7. Insall JN, Dorr LD, Scott RD et al (1989) Rationale of the Knee Society clinical rating system. Clin Orthop 248:13-14

8. Bellamy N, Buchanan W, Goldsmith J et al (1988) Validation study of WOMAC: a health status instrument for measuring clinically important patient relevant outcomes to antirheumatic drug therapy in patients with osteoarthritis of the hip or knee. J Rheumatol 15(12):1833-1840

9. Ware JE Jr, Sherbourne CD (1992) The MOS 36-item short-form health survey (SF-36). I. Conceptual framework and item selection. Med Care 30(6):473-483

10. Fehring TK, Odum S, Olekson C et al (2003) Stem fixation in revision total knee arthroplasty: a comparative analysis. Clin Orthop 416:217-224

11. Completo A, Fonseca F, Simões JA (2008) Strain shielding in proximal tibia of stemmed knee prosthesis: experimental study. J Biomech 41(3):560-566

12. Completo A, Simões JA, Fonseca F et al (2008) The influence of different tibial stem designs in load sharing and stability at the cement-bone interface in revision TKA. Knee 15(3):227-232

13. Burnett RS, Keeney JA, Maloney WJ et al (2009) Revision total knee arthroplasty for major osteolysis. lowa Orthop J 29:28-37

14. Jazrawi LM, Bai B, Kummer FJ et al (2001) The effect of stem modularity and mode of fixation on tibial component stability in revision total knee arthroplasty. J Arthroplasty 16(6):759-767

15. Skwara A, Figiel J, Knott T et al (2009) Primary stability of tibial components in TKA: in vitro comparison of two cementing techniques. Knee Surg Sports Traumatol Arthrosc 17(10):1199-1205

16. Cintra FF, Yepéz AK, Rasga MG et al (2015) Tibial component in revision of TKA: comparison between cemented and hybrid fixation. Rev Bras Ortop 46(5):585-590

17. Kim YH, Kim JS (2009) Revision total knee arthroplasty with use of a constrained condylar knee prosthesis. J Bone Jt Surg Am 91-A(6):1440-1447

18. Peters CL, Hennessey R, Barden RM et al (1997) Revision total knee arthroplasty with a cemented posterior-stabilized or constrained condylar prosthesis: a minimum 3-year and average 5-year follow-up study. J Arthroplasty 12(8):896-903

19. Mullaji A, Shetty GM (2014) Cemented stems: a requisite in revision total knee replacement. Bone Jt J 96-B(11 Suppl A):115-117

20. Baggio L, Gil-González S, Barra-González X et al (2016) Cemented stems in revision total knee arthroplasty on patients older than 75 y/o. Clinical and radiological analysis. Acta Ortop Mex 30(2):85-90

21. Whaley AL, Trousdale RT, Rand JA et al (2003) Cemented long-stem revision total knee arthroplasty. J Arthroplasty 18(5):592-599

22. Mabry TM, Vessely MB, Schleck CD et al (2007) Revision total knee arthroplasty with modular cemented stems: long-term follow-up. J Arthroplasty 22(6 Suppl 2):100-105
23. Jensen CL, Petersen MM, Schrøder HM, Lund B (2010) Changes in bone mineral dens of the distal femur after revision total knee arthroplasty with metaphyseal press-fit stem. J Orthop Traumatol 11(3):143-148

24. Greene JW, Reynolds SM, Stimac JD, Malkani AL, Massini MA (2003) Midterm results of hybrid cement technique in revision total knee arthroplasty. J Arthroplasty 28(4):570-574

25. Vince KG, Long W (1995) Revision knee arthroplasty: the limits of medullary fixation. Clin Orthop 317:172-177

26. Gofton WT, Tsigaras H, Butler RA et al (2002) Revision total knee arthroplasty: fixation with modular stems. Clin Orthop 404:158-168

27. Sah AP, Shukla S, Della Valle CJ et al (2011) Modified hybrid stem fixation in revision TKA is durable at 2 to 10 years. Clin Orthop 469(3):839-846

28. Shannon BD, Klassen JF, Rand JA et al (2003) Revision total knee arthroplasty with cemented components and uncemented intramedullary stems. J Arthroplasty 18(7 Suppl 1):27-32

29. Wood GC, Naudie DD, MacDonald SJ et al (2009) Results of press-fit stems in revision knee arthroplasties. Clin Orthop 467(3):810-817

30. Sheng PY, Konttinen L, Lehto M et al (2006) Revision total knee arthroplasty: 1990 through 2002. A review of the Finnish arthroplasty registry. J Bone Jt Surg (A) 88(7):1425-1430

31. Sheng PY, Jämsen E, Lehto M et al (2006) Revision total knee arthroplasty with the total condylar III system: a comparative analysis of 71 consecutive cases of osteoarthritis or inflammatory arthritis. Acta Orthop Sacand 77(3):512-518

32. Sheng P (2008) A study on revision total knee arthroplasty. Thesis. University of Tampere, Tampere

33. Gililland JM, Gaffney CJ, Odum SM et al (2014) Clinical \& radiographic outcomes of cemented vs. diaphyseal engaging cementless stems in aseptic revision TKA. J Arthroplasty 29(9 Suppl):224-228

34. Kosse NM, van Hellemondt GG, Wymenga AB et al (2017) Comparable stability of cemented vs press-fit placed stems in revision total knee arthroplasty with mild to moderate bone loss: 6.5-year results from a randomized controlled trial with radiostereometric analysis. J Arthroplasty 32(1):197-201

35. Wang C, Pfitzner T, von Roth P et al (2016) Fixation of stem in revision of total knee arthroplasty: cemented versus cementless - a meta-analysis. Knee Surg Sports Traumatol Arthrosc 24(10):3200-3211

36. Heesterbeek PJ, Wymenga AB, van Hellemondt GG (2016) No difference in implant micromotion between hybrid fixation and fully cemented revision total knee arthroplasty: a randomized controlled trial with radiostereometric analysis of patients with mild-to-moderate bone loss. J Bone Jt Surg Am 98(16):1359-1369

37. Haas SB, Insall JN, Montgomery W 3rd et al (1995) Revision total knee arthroplasty with use of modular components with stems inserted without cement. J Bone Jt Surg (Am) 77(11):1700-1707

38. Beckmann J, Lüring C, Springorum R et al (2011) Fixation of revision TKA: a review of the literature. Knee Surg Sports Traumatol Arthrosc 19(6):872-879

\section{Submit your manuscript to a SpringerOpen ${ }^{\circ}$ journal and benefit from:}

- Convenient online submission

Rigorous peer review

- Open access: articles freely available online

- High visibility within the field

Retaining the copyright to your article

Submit your next manuscript at springeropen.com 